\title{
SPONTANEOUS RESOLUTION OF A CASEOUS CALCIFICATION OF THE MITRAL ANNULUS
}

doi: $10.1590 / \mathbf{S 1 8 0 7 - 5 9 3 2 2 0 0 9 0 0 1 1 0 0 0 1 5}$

Michele Correale, ${ }^{\mathrm{I}}$ Giovanni Deluca, ${ }^{\mathrm{II}}$ Riccardo Ieva, ${ }^{\mathrm{I}}$ Matteo Di Biase ${ }^{\mathrm{I}}$

\section{INTRODUCTION}

An asymptomatic 76-year-old woman with a prior history of mitral annular calcification underwent a transthoracic echocardiographic examination for evaluation. Her risk factor for atherothrombotic disease was hypertension, and she had no prior medical history of hyperlipidemia or diabetes. She was given the following pharmacologic treatments: an angiotensin-converting enzyme (ACE) inhibitor (quinapril $5 \mathrm{mg}$ ), a calcium antagonist (lercanidipine $10 \mathrm{mg}$ ), a loop diuretic (furosemide $25 \mathrm{mg}$ ), and an antiplatelet agent (acetylsalicylic acid 150 $\mathrm{mg}$ ). A round, echo-dense structure was visualized in the left atrioventricular groove region (Fig. 1, upper panels).

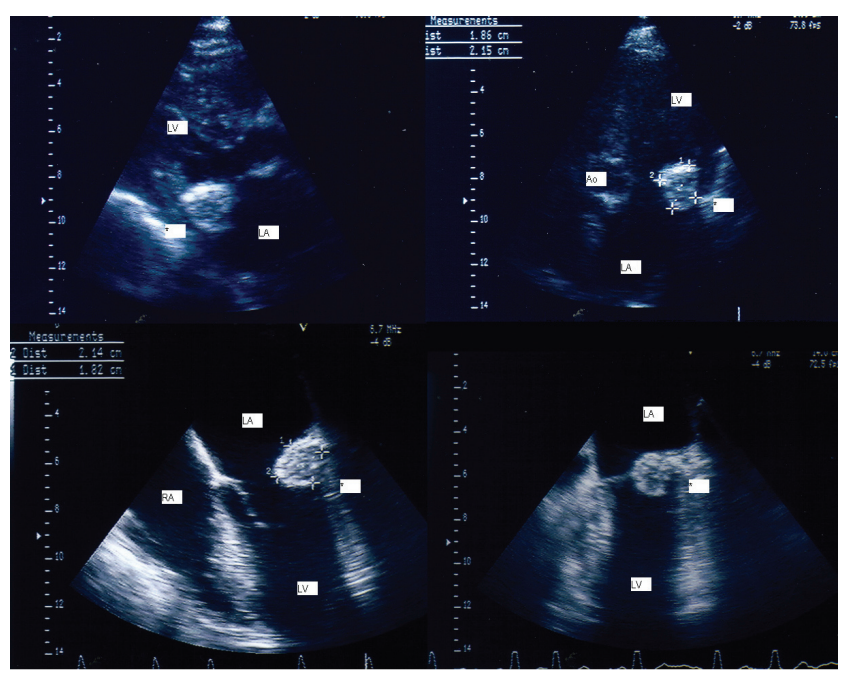

Figure 1 - Transthoracic two-dimensional visualization of the mass: parasternal long axis view and apical four-chamber view (upper panels). Transesophageal visualization of the mass (lower panels)

${ }^{\mathrm{I}}$ University of Foggia, Department of Cardiology - Foggia, Italy.

II Andria Hospital, Department of Cardiology - Andria, Italy.

Tel: 390881733652

Email: opsfco@tin.it
A transesophageal echocardiographic examination (TEE) was performed to better evaluate this intracardiac mass (Fig. 1, lower panels). A large $(2.14 \times 1.82 \mathrm{~cm})$, round, echo-dense mass containing central areas of echolucencies (central liquefaction) was revealed by TEE. No acoustic shadowing could be detected behind the mass, suggesting an absence of dense calcium deposition. The mass had sharp and distinct borders, with an echogenicity distinct from that of the adjacent myocardium (Fig. 2, left panel). The echocardiographic findings were consistent with the diagnosis of Caseous Calcification of the Mitral Annulus (CCMA). The patient was asymptomatic and was treated conservatively. A TEE conducted nine months later revealed the disappearance of the mass (Fig. 2, right panel).

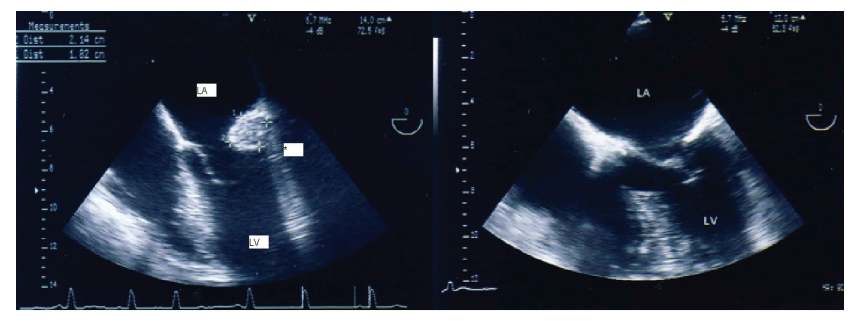

Figure 2 - Transesophageal visualization of the mass (left panel) and transesophageal examination repeated nine months later, showing the disappearance of the mass (right panel). LV: left ventricle; LA: left atrium; RA: right atrium; Ao: Aorta; *: Caseous calcification of the mitral annulus.

\section{DISCUSSION}

Mitral Annular Calcification (MAC) is a chronic degeneration of the mitral valve fibrous ring involving the posterior annulus. This disorder is common in the elderly, particularly in women. ${ }^{1}$ It may also occur in younger patients with advanced renal disease or other metabolic disorders that result in abnormal calcium metabolism. MAC is a consequence of atherosclerosis, with risk factors identical to those of cardiovascular disease. CCMA is a 
less-known and rarely described entity. This condition is an extensive "soft" periannular calcification, resembling a tumor, that is composed of an admixture of calcium, fatty acids, and cholesterol with a "toothpaste-like texture." CCMA is often misdiagnosed as a myocardial abscess,,$^{2,3}$ a tumor, ${ }^{4,5,6}$ or a thrombus, ${ }^{7}$ in some cases ${ }^{2}$ leading to unnecessary explorative cardiotomy. Differential diagnosis of round echogenic structures adjacent to the left atrioventricular groove should also include infected mitral calcification, lipomatosis of the atrioventricular groove, and enlarged lymph nodes. ${ }^{8}$ In this case, a correct diagnosis was made according to clinical presentation and echocardiographic findings. Generally, valvular endocarditis is unlikely in the absence of mitral valve regurgitation, fever, or positive blood cultures or laboratory examinations for infectious disease. Clinical signs of malignancy were absent. The round, echodense lobular aspect and mobility of the mass favored the diagnosis of myxoma; however, myxomas seldom originate from valves and are often found attached to the inter-atrial septum in the fossa ovalis region. Instead, specific echocardiographic features of CCMA favor the diagnosis: a large, round echodense mass in the posterior periannular mitral region that is heterogeneous and contains central echolucent areas of necrosis. The density of the mass suggested calcification and ruled out a thrombus diagnosis.

Harpaz and colleagues conducted a prospective series of echocardiographic examinations to define the echocardiographic appearance, prevalence, and prognosis of CCMA. ${ }^{9}$ They described the typical echocardiographic appearance of CCMA as a large, round, echo-dense mass with smooth borders situated in the periannular region, lacking acoustic shadowing artifacts, and containing central areas of echolucencies resembling liquefaction. They emphasized that it is possible to make a correct diagnosis by transthoracic examination alone, since no additional significant information was obtained by TEE in the 19 cases they examined. In this patient, however, TEE gave a more precise and detailed image of the mass and a better definition of the motion of the posterior mitral leaflet.

Recently, we ${ }^{10}$ have confirmed that CCMA is a rare and benign condition (14 patients with CCMA, $0.64 \%$ of all mitral annular calcifications, $0.068 \%$ of all studies). This study revealed no clinical characteristics typical of patients with CCMA. However, CCMA did tend to occur in older patients, and all 14 patients with CCMA had hypertension. We suggested that CCMA may be a dynamic process based on the observation that three patients with MAC progressed to CCMA and three patients with CCMA reverted back to MAC during the study period.

It is important to differentiate between CCMA and MAC; the former is a larger, well-delineated structure surrounded by clear borders with a distinct echolucency and a "softer" appearance that is less reflective and does not have acoustic shadowing. When a CCMA is perforated, a large amount of toothpaste-like, milky, caseous material is exuded from the mass. This material it is not truly calcific, but rather consists of caseous, putty-like material. After the material is removed, only a calcified envelope remains. In the case of large dense deposits of calcifications in the left atrioventricular groove, significant acoustic shadowing artifacts may rule out the diagnosis of CCMA. However, if the calcified envelope of the CCMA is very large, acoustic shadowing could result in an erroneous non-diagnosis of CCMA. ${ }^{10}$

The mechanisms involved in the liquefaction necrosis of CCMA are not well understood. Hypercholesterolemia and dissolution of lipid-laden macrophages might be implicated; high serum cholesterol is associated with MAC and may be responsible for liquefaction necrosis.

The peculiarity of this case is in its spontaneous resolution ten months later. A previous similar case ${ }^{11}$ in the literature showed a spontaneous resolution of a CCMA. In this previous case, the patient had a prior history of hemodialysis treatment, but no change was made in the hemodialysis regimen. In another similar case ${ }^{12}$ with a prior history of hemodialysis treatment, the reduction of the mass was observed after treatment with low calcium hemodialysis, so the authors suggested that the acute change in the serum calcium level might have contributed to the dissolution of the mass. In our case, we hypothesize that central liquefaction and dissolution of the material through a rupture of external wall occurred without evident clinical consequences.

In order to avoid misdiagnoses of CCMA as tumors, abscesses, or thrombi by echocardiographers, it is important that knowledge of this rare lesion becomes more widespread.

\section{REFERENCES}

1. Pomerance A. Pathological and clinical study of calcification of the mitral valve ring. J Clin Pathol. 1970;23:354-61.
2. Borowski A, Korb H, Voth E, de Vivie ER. Asymptomatic myocardial abscess. Thorac Cardiovasc Surg. 1988;36:338-40. 
3. Gilbert HM, Grodman R, Chung MH, Hartman G, Krieger KH, Hartman BJ. Sterile, caseous mitral valve "abscess" mimicking infective endocarditis. Clin Infect Dis. 1997;24:1015-6.

4. Teja K, Gibson RS, Nolan SP. Atrial extension of mitral annular calcification mimicking intracardiac tumor. Clin Cardiol. 1987;10:546-8.

5. Kautzner J, Vondracek V, Jrasel A, Belohlavek M. Tumor-like mitral annular calcification with central liquefaction. Echocardiography 1993;10:459-63.

6. Kato M, Nakatani S, Okazaki H, Tagusari O, Kitakaze M. Unusual appearance of mitral annular calcification mimicking intracardiac tumor prompting early surgery. Cardiology. 2006;106:164-6.

7. Stone E, Cohn D, Deal C, Pollock C. Calcific atrial mass in end-stage renal failure. Nephrol Dial Transplant. 1997;12:807-10.

8. De Conti F, Corbetti F, De Conti G, Mantovani E, Di Marco A, Zampiero A, et al. Unusual tumor-like calcification of the mitral annulus: diagnosis and tissue characterization by ultrasound, computed tomography and magnetic resonance imaging. Ital Heart J. 2005;6:160-3.
9. Harpaz D, Auerbach I, Vered Z, Motro M, Tobar A, Rosenblatt S. Caseous calcification of the mitral annulus: a neglected, unrecognized diagnosis. J Am Soc Echocardiogr. 2001;14:825-31.

10. Deluca G, Correale M, Ieva R, Del Salvatore B, Gramenzi S, Di Biase M. The incidence and clinical course of caseous calcification of the mitral annulus: a prospective echocardiographic study. J Am Soc Echocardiogr. $2008 ; 21: 828-33$

11. Gramenzi S, Mazzola AA, Tagliaferri B, Protasoni G, Brusoni D, d'Aloya G, et al. Caseous calcification of the mitral annulus: unusual case of spontaneous resolution. Echocardiography. 2005;22:510-3.

12. Koito H, Nakamura C, Suzuki J, Takahashi H, Iwasaka T. Reduced size of liquefaction necrosis of mitral annular calcification in chronic renal failure by using low calcium concentration hemodialysis. Jpn Circ J. 1999;63:490-2. 